\section{Prenatal exposure to the 1957 influenza epidemic}

SIR: Cannon et al (1996) conclude that the results of their case-control study of mothers suffering from influenza during pregnancy do not support the hypothesis that individuals exposed prenatally are at increased risk of schizophrenia. Although this conclusion is literally true, we believe that these negative results may well be due to a type 2 error. The authors have admitted that the study did not have sufficient power to investigate possible interactions, but they have failed to discuss the power to test the study hypothesis itself, and this appears to be extremely low. Crow (1994) has previously made this criticism of the same results when they were published in abstract form (Cannon et al, 1994), but the authors have failed to cite his editorial. They have also failed to cite a previous paper published by their own group which estimates that the excess risk associated with influenza exposure is in the order of $1.4 \%$ for every 1000 deaths in the two to three months before birth, and that such exposure usually accounts for $1-2 \%$ of all schizophrenic births (Sham et al, 1992). Another of their papers estimates the magnitude of the effect to be such that the 1957 epidemic (which produced a many-fold increase in influenza cases) resulted in only an $88 \%$ increase in schizophrenic births (O'Callaghan et al, 1991).

Cannon et al (1996) report 95\% confidence limits for the relative risk of schizophrenia due to influenza exposure as $0.41-2.95$. Although it is difficult to translate the previous estimates of effect size into a relative risk, it is clear that the latest results, far from being conclusively negative, are perfectly consistent with an effect much larger than any reported previously. It seems clear that their sample is much too small to stand much chance of producing definitive results. We estimate that with this sample there would need to have been nearly five times as many schizophrenic births in the exposed as opposed to the unexposed group in order to produce a statistically significant result. In fact, most previous studies have suggested that if there is any increased risk then it is confined to second-trimester exposure. If one restricted consideration to the 80 mothers exposed to influenza in the second trimester then one would need to observe a nine-fold increase in risk.

It is disheartening to realise that even such a carefully conducted study involving tracing hundreds of mothers and offspring actually stood very little chance of producing any conclusive result.
Cannon, M., Cotrer, D., ShaM, P. C., et al (1994) Schizophrenia in an Irish sample following prenatal exposure to the 1957 epidemic. Schizophrenia Research, 11, 95.

-, Cofrey, P., et al (1996) Prenatal exposure to the 1957 influenza epidemic and adult schizophrenia: a follow-up study. British Journal of Psychiatry, 168, 368-371.

Crow, T. J. (1994) Prenatal exposure to influenza as a cause of schizophrenia. British Journal of Psychiatry, 164, 588-592.

O'Callaghan, E., Sha从, P., TAXE, N., et al (1991) Schizophrenia after prenatal exposure to 1957 A2 influenza epidemic. Lancet 337, 1248-1250.

Sham, P. C., O'Callaghan, E., Take, N., et al (1992) Schizophrenia following prenatal exposure to influenza epidemics between 1939 and 1960. British Journal of Psychiatry, 160, 461-466.

M. WOODGATE D. Curtis

Royal London Hospital

London E1 $1 B B$

\section{Likelihood of hospital discharge}

SIR: Avnon \& Rabinowitz (1995) compared the rate of hospital discharge of patients remaining on clozapine with those who discontinued clozapine in a sample of 157 consecutively treated patients. As patients who failed to respond had clozapine discontinued by medical staff, the efficacy of clozapine compared with other treatments is exaggerated. It is therefore not surprising that patients who remained on clozapine were statistically more likely than those who discontinued to be successfully discharged from hospital.

We have compared the outcome of 15 patients who discontinued clozapine due to lack of efficacy or poor compliance and 16 who discontinued due to side-effects. The percentage of time spent in hospital, on average 2.7 years after first commencing clozapine, was significantly greater in the former group compared with those who discontinued due to side-effects $(78 \%$ v. $48 \%$, Mann-Whitney, $P<0.05)$.

While we agree that clozapine can be helpful for some patients with schizophrenia this paper does not clearly demonstrate clozapine's efficacy in increasing the likelihood of successful discharge.

Avnon, M. \& RaBnowrtz, J. (1995) Effectiveness of clozapine in hospitalised people with chronic neuroleptic-resistance. British Journal of Psychiatry, 167, 760-764.

R. DUFFETT

The Royal London Hospital (St Clements)

M. LAKER 